\title{
Aplicación de la metodología GARCH al precio de cierre en la Bolsa de Valores de Lima
}

\section{RESUMEN}

El artículo presenta una metodología que utiliza la serie de tiempos, para la predicción de los índices de los precios de cierre, que efectúan los centros de mercados bursátiles.El comportamiento actual responde a una expectativa generada sobre el valor de cambio producido en el momento precedente; es decir, a un valor esperado condicionado por la varianza del período anterior. El modelo GARCH es la parte fundamental de la investigación. Presenta de manera clara y detallada cada una de las actividades realizadas para la cuantificación del riesgo de mercado. Se aplica la metodología ARIMA para pronosticar los Rendimientos de la serie, que generalmente tienen una varianza no constante en el tiempo; es decir, presentan la existencia de heterocedasticidad y deben utilizarse los modelos autorregresivos generalizados de heterocedasticidad condicional, para la empresa en estudio.

Palabras clave: Predicción, series de tiempo, heterocedasticidad,modelos autorregresivos, metodología GARCH.

APPLICATION

OFGARCHMETHODOLOGYTO THE CLOSING PRICEON THELIMASTOCK EXCHANGE

\section{ABSTRACT}

The article presents a methodology that uses the time series, for forecasting indices closing prices, which made the stock market centers. The behavior response to a current generated on the expectation value of change in the preceding moment, ie an expected value conditioned by the variance of previous period. The GARCH model is the key part of the investigation. It presents a clear and detailed each of the activities undertaken to quantify market risk. ARIMA methodology is applied to predict the yields of the series, which generally have a variance is not constant over time, ie the existence of heteroscedasticity present and should be used generalized autoregressive conditional heteroskedasticity, for the company under study.

Keywords: Forecasting, time series heteroscedasticity, autoregressive models, GARCH methodology.

\section{SITUACION PROBLEMÁTICA}

En economía se habla de sucesos condicionados o de generación de expectativas a partir de los movimientos relativos que se han producido en el pasado. Lo usual es relacionar lo anterior, con las fuertes ondas en la evolución de las variables que, después de un gran sobresalto que dura más o menos días, tienden a retomar una senda de evolución tranquila.

En la teoría clásica de series temporales, el desarrollo estadístico se realiza a partir de un proceso estocástico estacionario; es decir de un proceso con: media constante, varianza constante y correlación entre dos observaciones distintas igual a la de otras dos cualquiera separadas por la misma distancia o mismo número de períodos (Anderson, 1999).

\section{ANTECEDENTES DE LA INVESTIGACIÓN}

Engle (1982) es el autor de una primera aproximación a la varianza condicional. Después de esto hay una amplia familia de sofisticaciones del modelo inicial que tienen nombres de modeIos GARCH, IGARCH, EARCH, TARCH, SWARCH, QS-ARCH, APARCH, FACTOR-ARCH.

El aspecto volátil está ligado con la medida del riesgo y los premios que busca un inversionista para los riesgos en sus activos. La modelación de pronósticos volátiles es en otras palabras la estructura de la covarianza del retorno de un activo.

El hecho que la volatilidad en los retornos fluctúa a lo largo del tiempo, es una realidad desde hace mucho tiempo. Originalmente perteneció al estudio de distribuciones leptocúrticas. Estos retornos han sido investigados por una serie de economistas en forma independiente.

En los trabajos de Mandelbrot (1963) y Mandelbrot y Taylor (1967) se aplicó la teoría de Pareto con la finalidad de caracterizar estas distribuciones de retorno. Rachev y Mittnik (2000) discuten estas distribuciones y su uso en la economía y las finanzas.

1 Ingeniero Industrial. Profesor del Departamento Académico de Ingeniería de Sistemas e Informática, UNMSM.

E-mail: eraffolecca@yahoo.es

2 Ingeniero Industrial. Profesor del Departamento Académico de Diseño y Tecnología Industrial, UNMSM.

E-mail: eraezg@unmsm.edu.pe

3 Ingeniero Industrial. Profesor del Departamento Académico de Ingeniería de Sistemas e Informática, UNMSM.

E-mail: cquispea@unmsm.edu.pe 
Las observaciones de serie de tiempos han sido modeladas usando la metodología $\mathrm{ARCH}$. El presente trabajo realiza su generalización usando GARCH.

\section{VOLATILIDAD}

La volatilidad es una medida de la velocidad del mercado, qué tan rápido se ajustan los precios de los activos financieros ante determinados hechos. Los mercados que se mueven despacio son mercados denominados de baja volatilidad, los mercados que se mueven rápidos son mercados denominados de alta volatilidad (Hamilton, 1994).

Muchos estudios realizados con referencia a los retornos de los activos, han mostrado evidencia de presencia de una volatilidad no constante en el tiempo. Al estudiar la relación dinámica entre las volatilidades de los rendimientos de los títulos se debe tener en cuenta la volatilidad y la covarianza asimétrica. El comportamiento asimétrico de la volatilidad hace referencia a la evidencia empírica según la cual un shock negativo sobre los rendimientos (caída inesperada del precio) conlleva a un aumento de la volatilidad mayor que un shock positivo sobre los rendimientos (aumento inesperado del precio) de la misma magnitud.

Cuando se refiere a volatilidad es importante distinguir entre dos formas de volatilidad: la volatilidad homocedástica y la volatilidad heterocedástica.La volatilidad homocedástica se refiere a la volatilidad que sería calculada como parámetro de una función de distribución de los rendimientos de un activo en el que se parte de la hipótesis de que la varianza de estos rendimientos no depende del tiempo sino que se mantiene constante.Por otro lado, la volatilidad heterocedástica se calcula tomando como punto de partida la hipótesis de desviación típica no constante en el tiempo, es un parámetro modelado por sí mismo (Nelson, 1991).

Es frecuente que en economía se hable de sucesos que estén condicionados con movimientos que se produjeron en el pasado, de manera especial en los mercados financieros con la estabilidad o inestabilidad de los mismos. Entonces es lógico definir a este tipo de variables que su comportamiento actual responde a una expectativa generada en el momento precedente, es decir al valor de volatilidad del periodo anterior.

La diferencia entre un suceso condicional y no condicional es que la expectativa condicional se refiere a una expectativa hacia el futuro pero sujeta a la información acumulada hasta el tiempo t. La no condicional no modifica el conjunto de información.
Las investigaciones en volatilidad fueron iniciadas por Robert Engle en 1982, quien propuso un proceso de volatilidad con varianza condicional en función del tiempo. Este proceso es denominado $\mathrm{ARCH}$, acrónimo de Autorregresivo con Heterocedasticidad Condicional (del inglés AutoregressiveConditionallyHeteroskedastic).

\section{MODELOS DE VOLATILIDAD ESTOCASTICA}

Un proceso estocástico univariante ${ }^{a}$ t sigue un modelo ARCH si y sólo si

$a_{t}=\sigma_{t} e_{t}$

Con $\sigma_{t}^{2}=\omega_{0}+\omega_{1} a_{t-1}^{2}$

$\omega_{0}>0, \omega_{1}>0, \omega_{1}<1$

Los modelos $A R C H$ relajan la hipótesis de la normalidad, y permiten que se tengan procesos de ruido blanco formados por variables dependientes. Es una clase de modelos con la propiedad:

$a_{t}=\sigma_{t} e_{t} e_{t} \sim N(0,1)$

Donde $\sigma_{\mathrm{t}} \mathrm{y} \theta_{\mathrm{t}}$ son dos procesos estacionarios independientes entre sí. El proceso $e_{t}$ es de ruido blanco normalizado, con media cero y varianza unitaria. El proceso $\sigma_{\mathrm{t}}$ es un proceso con estructura, con función al tiempo de los valores previos a t. La condición de independencia entre sí garantiza que la serie $a_{t}$ tenga media marginal igual a cero:

$\left.I E\left(a_{-t}\right)=E\left(\sigma_{t}\right]_{I}\right) E\left(e_{-t}\right)=0$

Y también la media condicional nula, ya que:

$\llbracket E\left(a 】 t \mid a_{\downarrow}(t-1) \dagger\right)=E\left(\sigma_{\downarrow} t+a_{\downarrow}(t-1)+\llbracket E(e \rrbracket \downarrow t)=0\right.$

Como el proceso $a_{t}$ es estacionario, tendrá una varianza marginal contante $\sigma^{2}$ :

$E\left(a_{1} t^{\dagger} 2\right)=E\left(\sigma_{1} t^{\dagger} 2\right) E\left(e_{1} t^{\dagger} 2\right)=E\left(\sigma_{1} t^{\dagger} 2\right)-=\sigma^{\dagger} 2$

Una varianza condicional que no es constante:

$\operatorname{Var}\left(a_{1} t^{\dagger} 2 \mid a_{l}(t-1) \downarrow\right)=E\left(\sigma_{1} t^{\dagger} 2 H \mid a_{1}(t-1)-\right) E\left(e_{1} t^{\dagger} 2\right)=\sigma_{1} t^{\dagger} 2$

El proceso $\sigma_{t}^{2}$ se interpreta como la varianza condicionada de la serie en cada instante, que varía en el tiempo con una estructura estacionaria.

Como $\sigma_{\mathrm{t}} \mathrm{y} e_{\mathrm{t}}$ son dos procesos estacionarios independientes entre sí; es garantía que $a_{t}$ carece de autocorrelación, y forma un ruido blanco. Las autocovarianzas de la serie $a_{t}$ son nulas: $I E\left(a_{t} a_{t-k}\right)=E\left(\sigma_{t} e_{t} \sigma_{t-k}\right)=E\left(\sigma_{t} \sigma_{t-k}\right) E\left(e_{t}\right)=0, k>0$

En contraste con las hipótesis de ARIMA, la serie $\sigma_{t}^{2}$ no es de variables independientes 
Los modelos ARCH generalizan las hipótesis de un ruido blanco, permitiendo dependencia, al hacer que la varianza condicional no sea constante.

La evidencia empírica muestra que un modelo ARCH de alto grado, se encuentra muy relacionada con la presencia dinámica de la varianza condicional. Esto es, realizar muchas evaluaciones para estimar los parámetros.

En 1986, Bollerslev propuso el modelo Generalizado Autorregresivo con Heterocedasticidad Condicional o GARCH, como una solución natural a los modelos ARCH de alto orden. Este modelo está basado en un $\mathrm{ARCH}$ de orden infinito. Reduciéndose de esta manera, el número de evaluaciones para la estimación de los parámetros.

Engle propuso un modelo para el proceso $\sigma_{t}^{2}$,

$\sigma_{t}^{2}=\omega_{0}+\sum_{i=1}^{p} \omega_{i} a_{t-i}^{2}$

$\operatorname{Con} \omega_{0}>0$ y $\omega_{i} \geq 0, i=1, \cdots, p$.

Considerando $\mathrm{ARCH}$ de primer orden un modelo $\mathrm{ARCH}(1)$, se tiene que:

$\sigma_{t}^{2}=\omega_{0}+\sum_{i=1}^{p} \omega_{i} a_{t-i}^{2}=\omega_{0}+\omega_{1} a_{t-1}^{2}$

Para el caso de varianza no condicional de $a_{t}$ se tiene:

$\sigma_{a}^{2}=E\left(a_{t}^{2}\right)=E\left(\omega_{0}+\omega_{1} a_{t-1}^{2}\right)=\omega_{0}+\omega_{1} \sigma_{a}^{2}$

$\sigma_{a}^{2}=\frac{\omega_{0}}{1-\omega_{1}}$

Con $\omega_{1}<1$ (Box, Jenkins y Reinsel, 2008).

En un proceso ARCH (p), se tiene que:

$\sigma_{t}^{2}=\omega_{0}+\sum_{i=1}^{p} \omega_{i} a_{t-i}^{2}=\omega_{0}+\omega_{1} a_{t-1}^{2}+\omega_{1} a_{t-2}^{2}+\cdots+\omega_{1} a_{t-p}^{2}$

$\sigma_{a}^{2}=E\left(a_{t}^{2}\right)=E\left(\omega_{0}+\omega_{1} a_{t-1}^{2}\right)=\omega_{0}+\omega_{1} \sigma_{a}^{2}+\cdots+\omega_{p} \sigma_{a}^{2}$

$\sigma_{a}^{2}=\frac{\omega_{0}}{1-\omega_{1}-\cdots-\omega_{p}}$

$\sum_{i=1}^{p} \omega_{i}<1$

El modelo ARCH (1) establece una dependencia con AR (1), al plantearse un proceso estocástico de los cuadrados de las observaciones, como un ruido blanco $v_{t}$ :

$v_{t}=a_{t}^{2}-\sigma_{t}^{2}$
Con media cero,

$E\left(v_{t}\right)=E\left(a_{t}^{2}\right)-E\left(\sigma_{t}^{2}\right)=0$

La varianza es una constante,

$\operatorname{var}\left(v_{t}\right)=E\left(a_{t}^{2}-\sigma_{t}^{2}\right)^{2}=E\left(a_{t}^{4}\right)+E\left(\sigma_{t}^{4}\right)-2 E\left(a_{t}^{2} \sigma_{t}^{2}\right)$

Se observa que:

$E\left(e_{t}^{4}\right)=3$

$E\left(a_{t}^{4}\right)=E\left(\sigma_{t}^{4} e_{t}^{4}\right)=3 E\left(\sigma_{t}^{4}\right)$

$E\left(a_{t}^{2} \sigma_{t}^{2}\right)=E\left(e_{t}^{2} \sigma_{t}^{2} \sigma_{t}^{2}\right)=E\left(\sigma_{t}^{4}\right)$

$\operatorname{var}\left(v_{t}\right)=2 E\left(\sigma_{t}^{4}\right)$

El proceso $v_{t}$ es de ruido blanco con media cero y varianza marginal constante.

Sea:

$\sigma_{t}^{2}=\omega_{0}+\omega_{1} a_{t-1}^{2}=-v_{t}+a_{t}^{2}$

Luego:

$a_{t}^{2}=\omega_{0}+\omega_{1} a_{t-1}^{2}+v_{t}$

Existe una dependencia similar al proceso AR (1) de los cuadrados.

\section{FAMILIA GARCH}

En los modelos GARCH, la varianza condicional es una función lineal de las tempranas varianzas condicionales calculadas.

En la generalización de $\mathrm{ARCH}$ o $\mathrm{GARCH}$, introducida por Bollerslev, se asume que el proceso $\sigma_{t}^{2}$ corresponde:

$\sigma_{t}^{2}=\omega_{0}+\sum_{i=1}^{p} \omega_{i} a_{t-i}^{2}+\sum_{i=1}^{q} \beta_{i} \sigma_{t-i}^{2}$

Con $\beta_{i} \geq 0, i=1, \cdots, q$.

GARCH se ha hecho popular y aceptado en el mundo de los pronósticos financieros, por los siguientes argumentos:

Está muy relacionado con los procesos ARMA; lo que sugiere que detrás de la teoría de $\mathrm{GARCH}$, se encuentra ARMA.

Es práctico efectuar un simple ajuste a los datos del mundo real, con sólo $\operatorname{GARCH}(1,1)$.

El modelo $\operatorname{GARCH}(1,1)$ para una serie de retornos $r_{\mathrm{t}}$ :

$r_{t}=\delta+a_{t}=\delta+\sigma_{t} e_{t}$

$\sigma_{t}^{2}=\omega_{0}+\omega_{1} a_{t-1}^{2}+\beta_{1} \sigma_{t-1}^{2}$ 
Para el caso de varianza no condicional de $a_{t}$ se tiene:

$$
\sigma_{a}^{2}=\frac{\omega_{0}}{1-\omega_{1}-\beta_{1}} \quad \text {,Siempre y cuando }
$$$$
\omega_{1}+\beta_{1}<1 \text {. }
$$

El modelo de Nelson (1991), modelo Exponencial GARCH o EGARCH, modela el logaritmo de la varianza condicional como:

$$
\mathbb{Z} \log \left(\sigma_{l}^{-2}\right)=\omega_{0}+\omega_{1}\left|\frac{a_{t-1}}{\sigma_{t-1}}\right|+\xi \frac{a_{t-1}}{\sigma_{t-1}}+\beta_{1} \log \left(\sigma_{t-1}^{2}\right)
$$

Una modificación de GARCH se debe a Glosten, Jagannathan y Runkle (1993), el GJR o TARCH (Threshold ARCH) que modela la varianza condicional para responder a los cambios de signos:

$$
I \sigma_{t}^{2}=\omega_{0}+\omega_{1} a_{t-1}^{2}\left[1-I_{\left\{a_{t-1}>0\right\}}\right]+\xi a_{t-1}^{2} I_{\left\{a_{t-1}>0\right\}}+\beta_{1} \sigma_{t-1}^{z}
$$

Donde ${ }^{I}\left\{a_{t-1}>0\right\}$ es 1 si es verdad que se cumple $\left\{a_{t-1}>0\right\}$.

Los modelos GARCH, capturan importantes características de una serie de tiempos en finanzas. De acuerdo a Engle (2004) una de las características en los rendimientos financieros de los activos de riesgo, corresponde a las colas pesadas (exceso de curtosis), y la otra corresponde a volatilidad agrupada o clúster de volatilidad de Mikosch, 2001; que traduce que a grandes cambios tienden a seguir grandes cambios; y pequeños cambios tienden a seguir pequeños cambios.

\section{DISCUSIÓN}

Los 5 años de Precios de Cierre Diario de la empresa en estudio, totalizan 1241 datos, como se presenta en la figura 1. Se observa que esta serie de tiempos es no estacionaria.

El proceso conocido como Análisis y Estimación, determina si los datos son heterocedásticos, estima los parámetros del modelo y desarrolla finalmente un análisis de post-estimación.

Si se dispone de la serie de tiempos, se denomina serie Precio, y se convierte a una serie Retorno o la variable Rentabilidad, usando la forma continua o periódica. La forma continua es de acuerdo a la ecuación:

$y_{t}=\frac{\log P_{t+1}}{P_{t}}=\log P_{t+1}-\log P_{t}$

El método continuo es el más utilizado en las series de tiempo financieras.
Figura 1. Serie de Precios de Cierre Diario de la empresa en estudio (2007-2011)

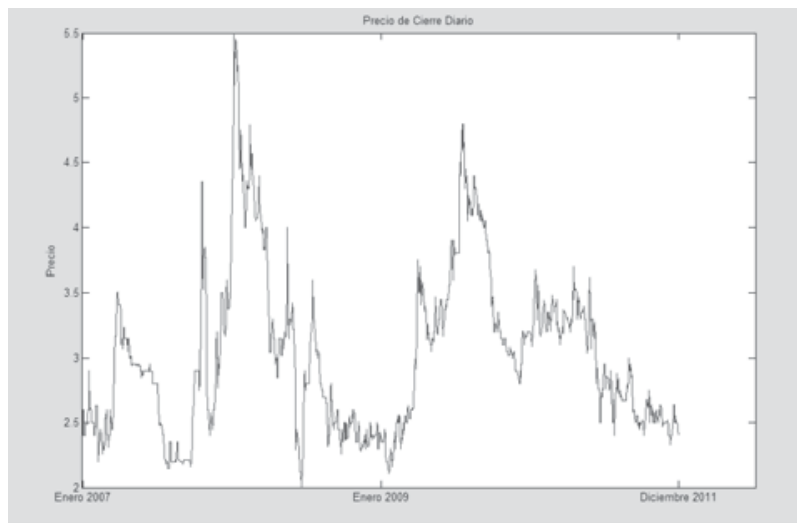

Fuente: Bolsa de Valores de Lima

El proceso conocido como Análisis y Estimación, determina si los datos son heterocedásticos, estima los parámetros del modelo y desarrolla finalmente un análisis de post-estimación.

Si se dispone de la serie de tiempos, se denomina serie Precio, y se convierte a una serie Retorno o la variable Rentabilidad, usando la forma continua o periódica. La forma continua es de acuerdo a la ecuación:

$y_{t}=\frac{\log P_{t+1}}{P_{t}}=\log P_{t+1}-\log P_{t}$

El método continuo es el más utilizado en las series de tiempo financieras.

La transformación Precio a Retorno, tiene como finalidad generar una data estable para la modelación GARCH.

En la figura 2, se presenta la serie de Retorno ajustada en forma continua, esta es de tipo estacionaria, que es supuesto típico en los modelos GARCH. La transformación Precio a Retorno, tiene como finalidad generar una data estable para la modelación GARCH.

Se observa que la Rentabilidad para los periodos estables oscila en la banda de $\pm 3 \%$; y los periodos en los cuales la volatilidad claramente se sale de dicho rango y oscila hasta en $\pm 15 \%$.

En la figura 3, se observa que el valor del rendimiento promedio es casi cero, es decir que en el mercado peruano para la empresa en estudio, se mueve en torno a cero, por lo que en promedio no hay ni pérdidas ni ganancias.

Una simple observación de la gráfica se puede afirmar a priori que en el centro de la densidad de probabilidades es menor con relación a la normal, en tanto que los extremos de la distribución particu- 
larmente a la izquierda, se concentra una densidad de probabilidades mayor en relación también a lo normal.

Figura 2. Serie de Retorno basado en Precio de Cierre Diario

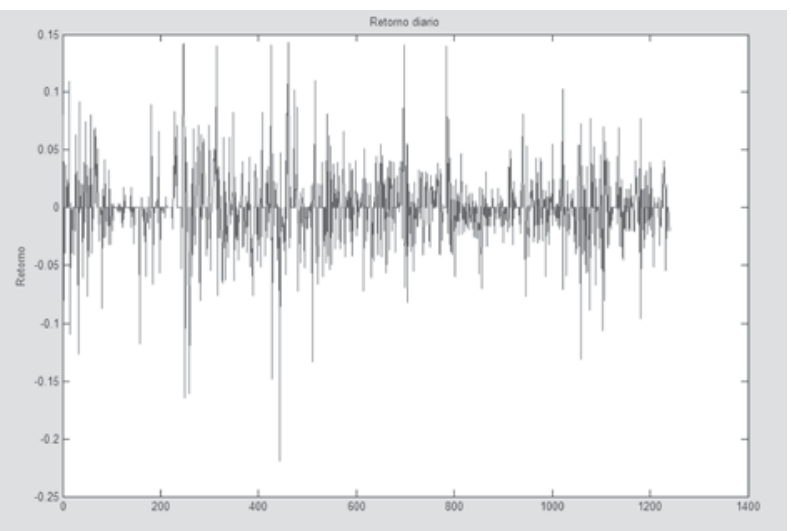

Figura 3. Histograma y estadísticos para la Rentabilidad

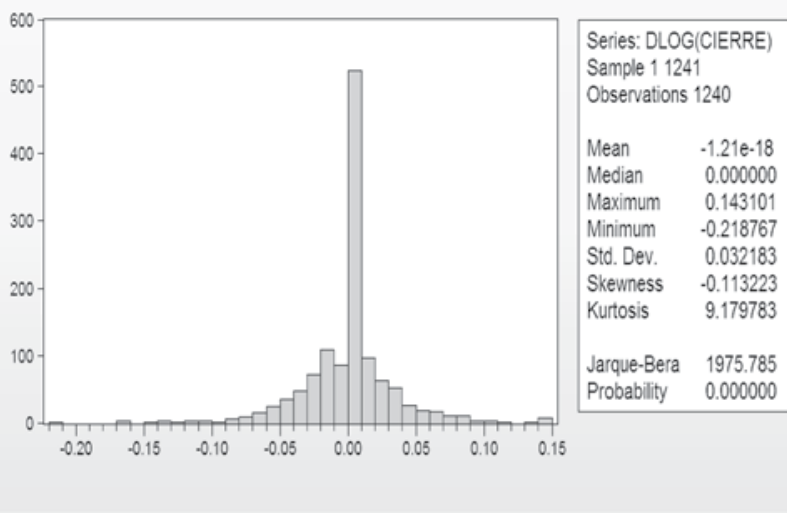

La ausencia de la normalidad se demuestra por la curtosis que es de 9.179783 , muy superior a 3 que es una distribución estándar; el valor de la asimetría es - 0.113223 es levemente inferior a la distribución normal que es 0 ; indicando una cola izquierda mayor que la normal. Finalmente el estadístico JarqueBera alcanza un valor de 1975.785, muy alto. Todo ello nos lleva a rechazar la hipótesis de normalidad.

La distribución de la Rentabilidad o de Retornos posee leptokurtosis.

Un análisis de estacionariedad conlleva a realizar una prueba estadística para determinar la existencia de Raíces Unitarias mediante el test DickeyFuller Ampliado o DFA. Desde la tabla 1 se observa que al $1 \%, 5 \%$ y $10 \%$ de confianza se rechaza la hipótesis nula, por lo tanto no existe evidencia estadística que la serie de Rentabilidad es estacionaria. También se observa que en el test Durbin-Watson o DW, los rezagos incorporados en el test DFA eliminaron correctamente posibles problemas de autocorrelación en los residuos.
Tabla 1. Test de Dickey-Fuller Ampliado

\begin{tabular}{|c|c|c|c|}
\hline Estadístico & $\begin{array}{c}\text { Con } \\
\text { intercepto }\end{array}$ & $\begin{array}{c}\text { Tendencia } \\
\text { Con intercepto }\end{array}$ & Ninguno \\
\hline $\begin{array}{c}1 \% \text { valor } \\
\text { crítico }\end{array}$ & -3.435415 & -3.965470 & -2.566830 \\
\hline $\begin{array}{c}5 \% \text { valor } \\
\text { crítico }\end{array}$ & -2.863664 & -3.413442 & -1.941079 \\
\hline $\begin{array}{c}10 \% \text { valor } \\
\text { crítico }\end{array}$ & -2.567951 & -3.128762 & -1.616527 \\
\hline DW & 1.997195 & 1.997098 & 1.997185 \\
\hline
\end{tabular}

El chequeo de la correlación se consigue evaluando la correlación entre un rango de desplazamientos o Lag. Se observa en la figura 4 la función de la autocorrelación o ACF. En la gráfica se encuentra que para el Lag igual a cero es 1 . Los límites de ACF se encuentran entre -0.0568 y 0.0568 .

Figura 4. ACF con límites para la serie de Retornos

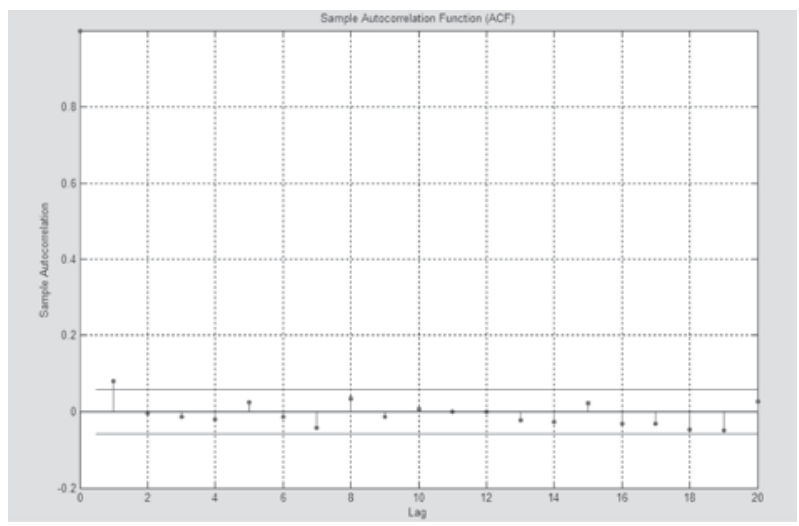

Similarmente el chequeo de la correlación se consigue evaluando la correlación parcial entre un rango de desplazamientos o Lag. Se observa en la figura 5 la función de la autocorrelación parcial o PACF. En la gráfica se encuentra que para el Lag igual a cero es 1. Los límites de PACF se encuentran entre -0.0568 y 0.0568 .

Figura 5. PACF con límites para la serie de Retornos

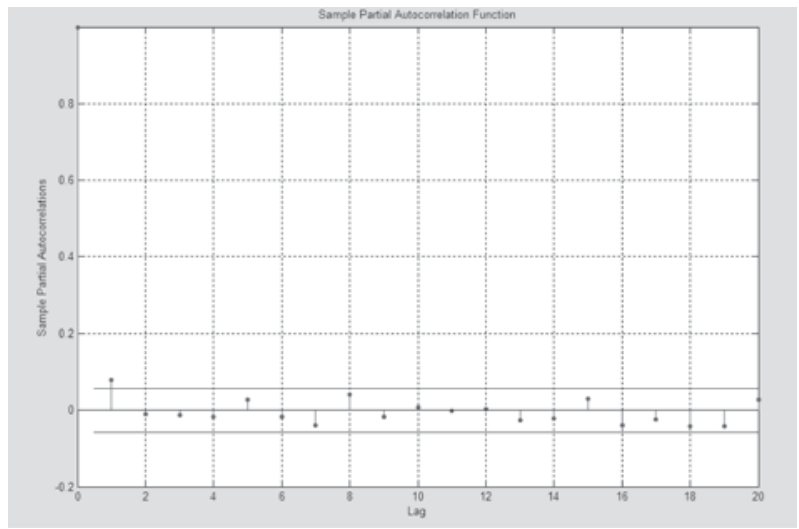


En la figura 6 se presenta la correlación del cuadrado de la serie Retorno. Se observa que existe la posibilidad un proceso de varianza cerrado a la no estacionalidad.

Figura 6. ACF del cuadrado de los Retornos

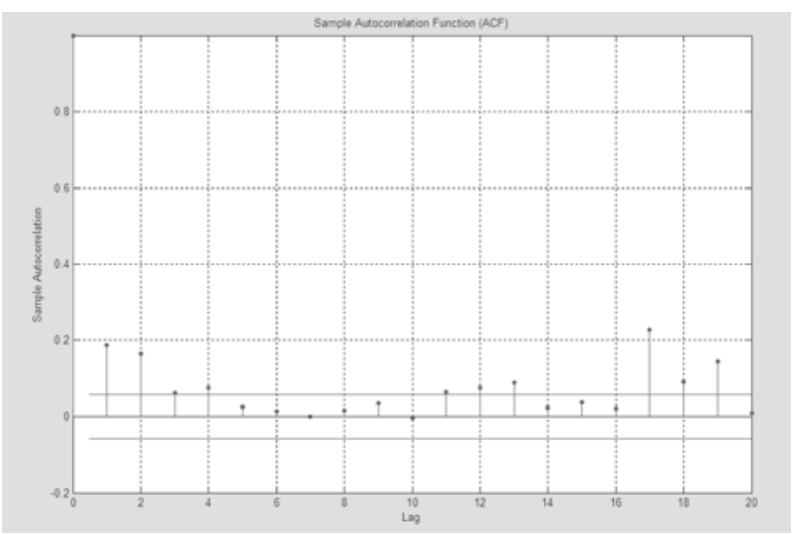

Para cuantificar la correlación se utiliza como prueba de hipótesis, el test de Q Ljung-Box-Pierce y el de ARCH Engle.

El valor de la hipótesis $\mathrm{H}$ es 0 cuando no hay significancia en la correlación (no rechaza la hipótesis nula); si H es 1 cuando hay significancia en la correlación (se rechaza la hipótesis nula).

Se observa en el test $Q$ que no hay correlación significante para valores de Lag a un nivel de 0.05 de significancia.

00.209213 .265418 .3070

00.424315 .381924 .9958

00.235024 .171931 .4104

Sin embargo si hay significancia en la correlación cuando se trata de la serie de los cuadrado del Retorno.

\subsection{0 .000092 .455618 .3070 \\ 1.00000116 .952524 .9958}

1.00000220 .040731 .4104

El test ARCH de Engle, muestra la evidencia de la significancia en el efecto GARCH; es decir la heterocedasticidad.

$$
\begin{aligned}
& 1.00000 .000069 .731218 .3070 \\
& 1.00000 .000079 .213524 .9958 \\
& 1.00000151 .903531 .4104
\end{aligned}
$$

El modelo $\operatorname{GARCH}(1,1)$ para la serie de retornos $y_{\mathrm{t}}$ es:

$y_{t}=\delta+a_{t}$

$\sigma_{t}^{2}=\omega_{0}+\omega_{1} a_{t-1}^{2}+\beta_{1} \sigma_{t-1}^{2}$
Usando los valores obtenidos:

$\delta=C=-0.00078447$

$\omega_{0}=K=4.6307 \times 10^{-5}, \omega_{1}=0.075585, \beta_{1}=0.87995$

Se obtiene la estimación:

$y_{t}=-0.00078447+a_{t}$

$\sigma_{t}^{2}=4.6307 \times 10^{-5}+0.075585 a_{t-1}^{2}+0.87995 \sigma_{t-1}^{2}$

Siendo el valor de log verosimilitud LLF $=2582.61$.

Las relaciones derivadas desde el ajuste del modelo, residuales o innovaciones, las desviaciones estándares condicionales y el retorno observado son presentados en la figura 7 . Se observa que el hecho de ser la suma de los coeficientes menor que 1 , es una condición de ser cerrada a la condición de la no estacionariedad:

$\omega_{1}+\beta_{1}=0.955535<1$

Las innovaciones o residuales son las diferencias entre los Retornos y el ajuste del modelo. Las desviaciones estándar corresponden a $\sigma_{\mathrm{t}}$. En la figura 7 se muestra la volatilidad conglomerada o clúster del Retorno.

Figura 7. Errores estándares

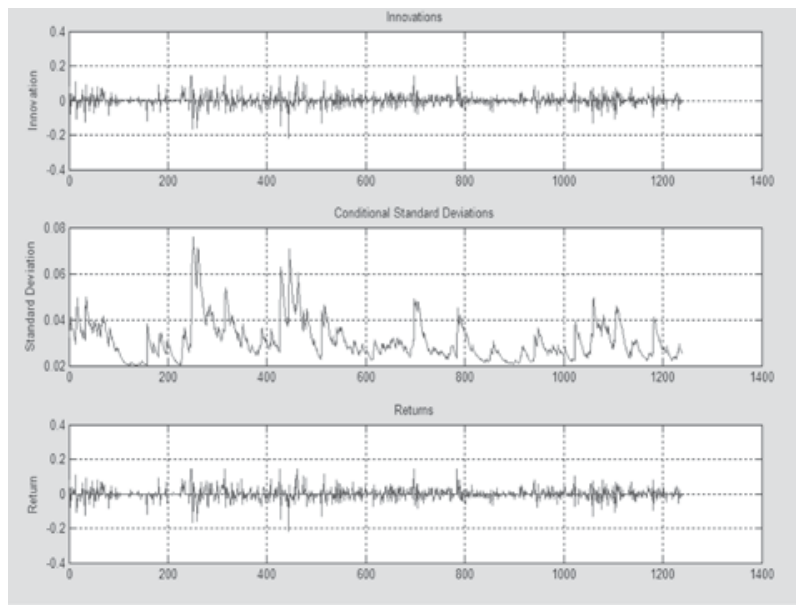

Las relaciones derivadas desde el ajuste del modelo, residuales o innovaciones, las desviaciones estándares condicionales y el retorno observado son presentados en la figura 7 . Se observa que el hecho de ser la suma de los coeficientes menor que 1 , es una condición de ser cerrada a la condición de la no estacionariedad:

$\omega_{1}+\beta_{1}=0.955535<1$

Las innovaciones o residuales son las diferencias entre los Retornos y el ajuste del modelo. Las desviaciones estándar corresponden a $\sigma_{\mathrm{t}}$. En la gráfica se muestra la volatilidad conglomerada o clúster del Retorno. 
Desde la expresión $a_{t}=\sigma_{t} \epsilon_{t}$, se obtiene $\epsilon_{t}$ como la relación de las innovaciones con respecto a la desviación estándar condicional $\bar{e}_{\mathrm{t}}=\frac{\mathrm{a}_{\mathrm{t}}}{\sigma_{\mathrm{t}}}$. Estas innovaciones denominadas estandarizadas se presentan en la figura 8 . Se observa que los clúster son pequeños y estables.

Figura 8. Innovaciones estandarizadas

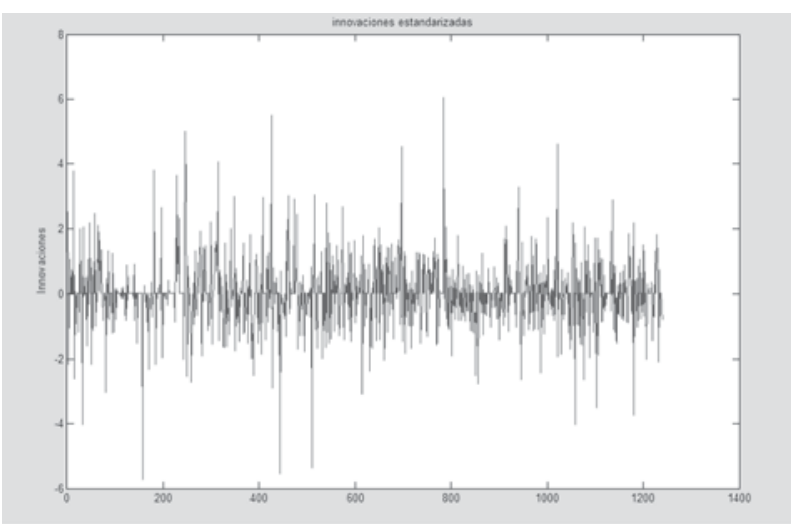

En la figura 9, se presenta la función ACF para el cuadrado de las innovaciones estandarizadas. Se observa la presencia de la heterocedasticidad en los retornos.

Figura 9. ACF de las innovaciones estandarizadas al cuadrado

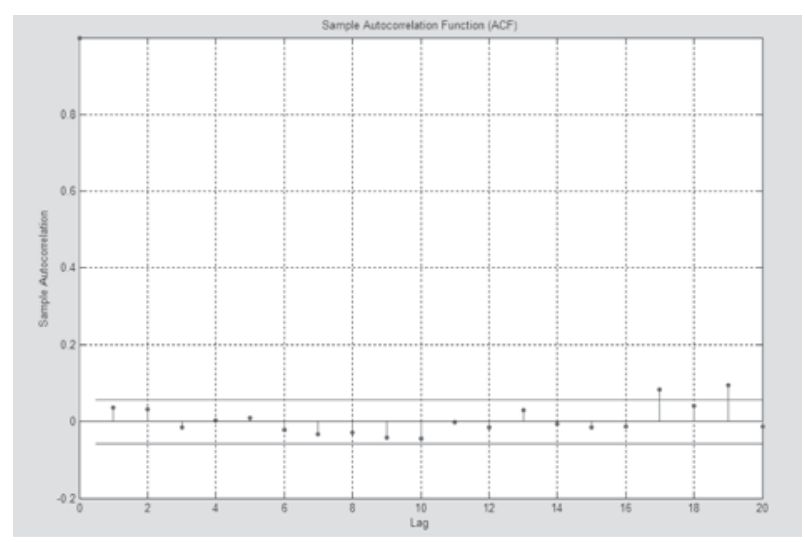

Comparando desde el test $Q$ y el de $A R C H$ con los resultados de la Pre-Estimación; se observa que desde el cuadrado de las innovaciones estandarizadas, en el test $Q$ que no hay correlación significante para valores de Lag a un nivel de 0.05 de significancia (salvo el caso de Lag20).

$$
00.360810 .958118 .3070
$$$$
00.620812 .760424 .9958
$$

$$
1.00000 .018435 .336131 .4104
$$

En el caso del test de Engle, se tiene que no hay correlación significante para valores de Lag a un nivel de 0.05 de significancia.
A partir del modelo por defecto $\operatorname{GARCH}(1,1)$, en esta sección se plantean otros modelos alternativos de GARCH.

Para la selección y análisis de los modelos, esta sección hace uso de las siguientes herramientas:

- Pruebas del ratio de verosimilitud.

- Criterios de Información de Akaike y el Bayesiano.

Un criterio alternativo para encontrar el ajuste de un modelo que minimiza los errores residuales, corresponde a los métodos de la Máxima Verosimilitud o ML (MaximumLikelihood). En estos métodos se encuentran los parámetros, maximizando la probabilidad de la ocurrencia de la data observada.

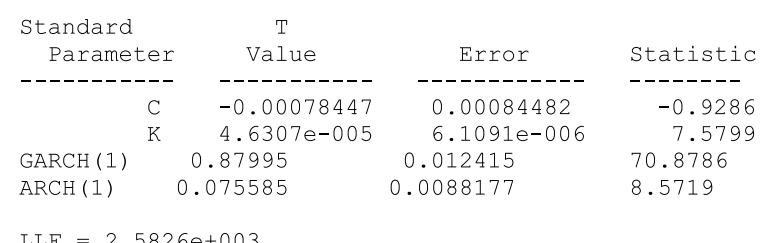

El modelo $\operatorname{GARCH}(2,1)$ para la serie de retornos yt es:

$y_{t}=\delta+a_{t}$

$\sigma_{t}^{2}=\omega_{0}+\omega_{1} a_{t-1}^{2}+\beta_{1} \sigma_{t-1}^{2}+\beta_{2} \sigma_{t-2}^{2}$

En el caso del modelo GARCH (2, 1): $y_{t}=-0.00082608+a_{t}$

$\sigma_{t}^{2}=5.5046 \times 10^{-5}+0.089422 \alpha_{t-1}^{2}+0.60004 \sigma_{t-1}^{2}+0.25692 \sigma_{t-2}^{2}$

\begin{tabular}{rllr} 
Parameter & \multicolumn{1}{c}{ Value } & \multicolumn{1}{c}{ Error } & Statistic \\
- C & -0.00082608 & 0.00085196 & -0.9696 \\
K & $5.5046 e-005$ & $1.1605 e-005$ & 4.7433 \\
GARCH (1) & 0.60004 & 0.2277 & 2.6353 \\
GARCH (2) & 0.25692 & 0.20543 & 1.2507 \\
ARCH (1) & 0.089422 & 0.016587 & 5.3912
\end{tabular}

$\mathrm{LLF}=2.5836 \mathrm{e}+003$

Desde la tabla 2, se presenta el resumen de los valores LLF para el modelo por defecto y una alternativa. El modelo $\operatorname{GARCH}(2,1)$ es más preciso con valor de 2583.6.

Tabla 2. Aplicación del LRT

\begin{tabular}{|c|c|}
\hline Modelo & LLF \\
\hline $\operatorname{GARCH}(\mathbf{1}, \mathbf{1})$ & 2582.6 \\
\hline $\operatorname{GARCH}(\mathbf{2}, \mathbf{1})$ & 2583.6 \\
\hline
\end{tabular}

Efectuando la prueba del ratio de verosimilitud o LRT (Likelihood Ratio Test); donde $\operatorname{GARCH}(1,1)$ sirve como hipótesis nula y $\operatorname{GARCH}(2,1)$ como una hipótesis alternativa, se determina:

00.168881 .89293 .8415 
Se encuentra que $\mathrm{H}=0$, indica que existe insuficiente evidencia estadística para utilizar el modelo GARCH $(2,1)$. En otras palabras el modelo GARCH $(1,1)$ explica adecuadamente la variabilidad en la serie de retornos, cuando se le compara con un modelo más elaborado como es el modelo GARCH $(2,1)$. Esta prueba está realizada con un nivel de significancia de $\alpha=0.5$; pValue 0.16888 por el que se rechaza la hipótesis nula; Criticalvalue es el valor crítico de la distribución de chi-cuadrado; y Ratio es evaluado por la expresión:

Ratio $=2(L L F 21-L L F 11)$

Dónde:

$\operatorname{LLF} 21=\operatorname{LLF}$ de GARCH $(2,1), \operatorname{LLF} 11=\operatorname{LLF}$ de $\operatorname{GARCH}(1,1)$

Una de las formas para la selección de un modelo, es mediante el uso del Criterio de Información; tales como AIC propuesto por Akaike o el Bayesiano (BIC) de Schwarz.

Para efectuar los criterios AIC y BIC es necesario conocer el número de parámetros o coeficientes de cada modelo: $\operatorname{GARCH}(2,1)$ posee 5 argumentos: $r=p+q+2$ (los dos coeficientes son por $\mathrm{C}$ y $\mathrm{K}$ ); en cambio $\operatorname{GARCH}(1,1)$ posee sólo 4 coeficientes.

Tabla 3. Criterios de Información

\begin{tabular}{|c|c|c|}
\hline Modelo & AIC & BIC \\
\hline $\operatorname{GARCH}(1,1)$ & -5157.212312029322 & -5134.808702191154 \\
\hline $\operatorname{GARCH}(2,1)$ & -5157.105193880294 & -5129.100681582584 \\
\hline
\end{tabular}

Los criterios corresponden a las siguientes expresiones:

$$
\begin{aligned}
& A I C=(-2 L L F)+(2 r) \\
& B I C=(-2 L L F)+(r \cdot \log (\text { Num.observaciones }))
\end{aligned}
$$

Desde la tabla 3 que contiene los criterios de información expuetos, el criterio AIC favorece tanto al modelo $\operatorname{GARCH}(2,1)$ como $\operatorname{GARCH}(1,1)$ por el valor menor, que para el caso son iguales; y el criterio BIC favorece al modelo $\operatorname{GARCH}(1,1)$.

La modelación con ARCH y GARCH, se compone de dos ecuaciones: una para la media condicional y otra para la varianza condicional.

La ecuación de la media: $y_{t}=\delta+a_{t}+A R_{1} y_{t-1}$

La ecuación de la varianza para el proceso $\mathrm{ARCH}$ (1): $\sigma_{t}^{2}=\omega_{0}+\omega_{1} a_{t-1}^{2}$

La ecuación de la varianza para el proceso GARCH $(1,1): \sigma_{t}^{2}=\omega_{0}+\omega_{1} a_{t-1}^{2}+\beta_{1} \sigma_{t-1}^{2}$

La ecuación de la varianza para el proceso GARCH $(p, q)$ :

$\sigma_{t}^{2}=\omega_{0}+\sum_{i=1}^{p} \omega_{i} a_{t-i}^{2}+\sum_{i=1}^{q} \beta_{i} \sigma_{t-i}^{2}$

Desde la tabla 4 , se descarta $\operatorname{GARCH}(2,1)$ y $\operatorname{GARCH}(2,2)$ por tener coeficientes negativos.

Tabla 4. Coeficientes para los modelos ARCH/GARCH

\begin{tabular}{|c|c|c|c|c|c|c|c|}
\hline \multirow{2}{*}{ Modelo } & \multicolumn{2}{|c|}{ Ecuación de la media } & \multicolumn{5}{c|}{ Ecuación de la varianza } \\
\cline { 2 - 8 } & C & AR1 & C & A1 & G1 & A2 & G2 \\
\hline ARCH(1) & -0.000857 & 0.035817 & 0.000827 & 0.188837 & & & \\
\hline GARCH(1,1) & -0.000790 & 0.030652 & 0.0000468 & 0.074435 & 0.880110 & & \\
\hline GARCH(1,2) & -0.000833 & 0.025771 & 0.0000552 & 0.087968 & 0.593334 & & 0.26429 \\
\hline GARCH(2,1) & -0.000943 & 0.016163 & 0.0000288 & 0.133247 & 0.92460 & -0.8733 & \\
\hline GARCH(2,2) & -0.000925 & 0.031865 & 0.000007 & 0.137566 & 1.475202 & -0.1249 & -0.49584 \\
\hline
\end{tabular}


Tabla 5. Coeficientes de Akaike y Schwarz

\begin{tabular}{|c|c|c|}
\hline \multirow{2}{*}{ Modelo } & \multicolumn{2}{|c|}{ Coeficientes } \\
\cline { 2 - 3 } & AIC & BIC \\
\hline ARCH(1) & -4.103162 & -4.086626 \\
\hline GARCH $(1,1)$ & -4.162552 & -4.141882 \\
\hline GARCH $(1,2)$ & -4.162507 & -4.137702 \\
\hline
\end{tabular}

Desde la tabla 5 , se encuentra que $\operatorname{GARCH}(1,1)$ posee menores valores en AIC y BIC.

El modelo $\operatorname{GARCH}(1,1)$ queda definido por:

$$
\begin{aligned}
& y_{t}=-0.000790+a_{t}+0.030652 y_{t-1} \\
& \sigma_{t}^{2}=4.68 \times 10^{-5}+0.074435 a_{t-1}^{2}+0.880110 \sigma_{t-1}^{2}
\end{aligned}
$$

Los mercados financieros tienen como característica que ante malas noticias se producen caídas en las cotizaciones que tienen una volatilidad mayor que cuando se producen subidas en las cotizaciones por buenas noticias, en tal caso las volatilidades son de menor magnitud.

Estos casos denominados de volatilidad simétrica, fueron el origen de los modelos TARCH y EGARCH. La ecuación de la media para el proceso TARCH $(1,1)$ :

$y_{t}=\delta+a_{t}+A R_{1} y_{t-1}$

La ecuación de la varianza para el proceso TARCH $(1,1)$ :

$\sigma_{t}^{2}=\omega_{0}+\omega_{1} a_{t-1}^{2}+\beta_{1} \sigma_{t-1}^{2}+\gamma_{1} a_{t-1}^{2} d_{t-1}$

El modelo TARCH $(1,1)$, tiene como AIC el valor de -4.174201 y BIC el valor -4.149397 . El criterio de información de Akaike para GARCH $(1,1)$ con valor de -4.162552, luego se decide al TARCH $(1,1)$.

El modelo TARCH $(1,1)$ está representado como:

$$
\begin{aligned}
& \text { La ecuación de la media } \\
& y_{t}=-5.87 \times 10^{-5}+a_{t}+0.020683 y_{t-1}
\end{aligned}
$$

La ecuación de la varianza para el proceso:

$\sigma_{t}^{2}=3.96 \times 10^{-5}+0.101464 a_{t-1}^{2}+0.895861 \sigma_{t-1}^{2}-0.073233 a_{t-1}^{2} d_{t-1}$

El modelo EGARCH $(1,1)$ se encuentra que la ecuación de la media es cero, de la misma forma su R-squared y su ajustado no tienen significación. Luego no se considera para el análisis este modelo.
El modelo CGARCH $(1,1)$, tiene como AIC el valor de -4.172131 y BIC el valor -4.139058 . El criterio de información de Akaike para TARCH $(1,1)$ es con valor de -4.174201. El menor AIC es de TARCH $(1,1)$.

$m_{t}=0.000978+0.977213\left(m_{t-1}-0.000978\right)+0.026958\left(a_{t-1}^{2}-\sigma_{t-1}^{2}\right)$ $\sigma_{t}^{2}-m_{t}=-0.033838\left(a_{t-1}^{2}-m_{t-1}\right)+0.495980\left(\sigma_{t-1}^{2}-m_{t-1}\right)$

En la figura 10 presenta la gráfica de la desviación condicional de la regresión de $\operatorname{TARCH}(1,1)$.

Figura 10. Gráfica de la desviación estándar condicional para el modelo TARCH $(1,1)$

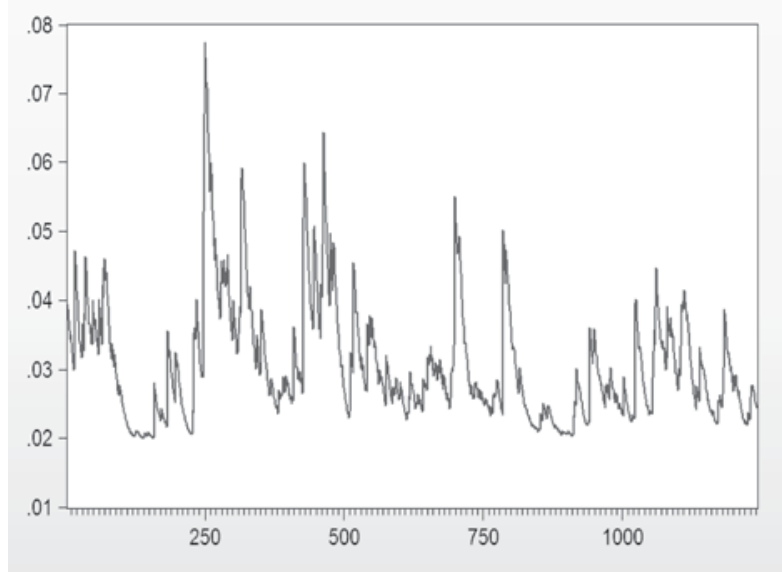

El resultado de los análisis y regresiones tratados en este trabajo, lleva a la conclusión de que el modelo más adecuado para la estimación y predicción de la volatilidad de los precios de cierre de la empresa del estudio es el modelo TARCH $(1,1)$, toda vez que es quién ofrece mejores respuestas en todos los horizontes.

\section{CONCLUSIONES}

1. Se utilizaron los modelos de la familia $\mathrm{ARCH}$, en el contexto de la predicción de la volatilidad.

2. La serie de Rendimiento evidenciaron un exceso de curtosis, validando que las distribuciones de colas pesadas, son parte de los precios de cierre analizados.

3. Se detectaron clúster de volatilidad, razón para que la familia ARCH sea la más adecuada a utilizar.

4. El análisis siguiere que la volatilidad es heterocedástica.

5. El resultado de los análisis y regresiones realizadas, conlleva a la conclusión de que el modelo más adecuado para la estimación y predicción de la volatilidad del Rendimiento es el modelo TARCH $(1,1)$, ya que es quién ofrece mejores predicciones en el horizonte. 


\section{REFERENCIAS BIBLIOGRÁFICAS}

[1] ANDERSON, D., SWEENEY, D., WILLIAMS, T. (1999). "Estadística para Administración y Economía", International Thompson Editores.

[2] BOLLERSLEV, T., "Generalized Autoregressive Conditional Heteroskedasticity", JOURNAL OF ECONOMETRICS, 31, 1986.

[3] BOLLERSLEV, T., "Glossary to ARCH (GARCH)", CREATES, RESEARCH PAPER 2008-49, 2008.

[4] box, g., jenkins, g., reinsel, g. (2008). Time Series Analysis: Forecasting and Control. New Jersey: john wiley\& sons, fourth edition.
[5] engle, r. (1982). Autoregressive Conditional Heteroskedasticity with Estimates of the Variance of U.K. Inflation, econometrica, 50.

[6] glosten, l., jagannathan, r. y runkle, d. (1993), "On the Relation Between the Expected Value and the Volatility of the Normal Excees Return on Stocks", journal of finance, 48.

[7] hamilton, j., "Time Series Analysis", princeton university press, 1994.

[8] NELSON, D., "Conditional Heteroskedasticity in Asset Returns: A New Approach", ECONOMETRICA, 59, 1991. 